\title{
Channel-Aware Energy Allocation for Throughput Maximization in Massive Low-Rate Multiple Access
}

\author{
Francesc Molina, Josep Sala-Álvarez, Francesc Rey, and Javier Villares \\ Signal Theory and Communications Department, Technical University of Catalonia (UPC) \\ E-mail: \{francesc.molina, josep.sala, francesc.rey, javier.villares\}@upc.edu
}

\begin{abstract}
A multiple access (MA) optimization technique for massive low-rate direct-sequence spread spectrum communications is analyzed in this work. A dense network of users transmitting at the same rate to a common central node under channelaware energy allocation is evaluated. At reception, successive interference cancellation (SIC) aided by channel decoding is adopted. Our contribution focuses on wireless scenarios involving a vast number of users for which the provided user-asymptotic model holds. Variational calculus (VC) is employed to derive the energy allocation function that, via user-power imbalance, maximizes the network spectral efficiency (SE) when perfect channel state information at transmission (CSIT) is available and both average and maximum per-user energy constraints are set. Monte Carlo simulations at chip-level of a SIC receiver using a real decoder assess the proposed optimization method.
\end{abstract}

Index Terms-Successive interference cancellation, massive multiple access, packet error rate, low-rate communications

\section{INTRODUCTION}

Massive access communications have gained a lot of interest due to consumer traffic growth [1]. In the near future, a large number of energy-aware devices will exchange short payload data. The way to manage such high interference levels is challenging the performance of network MA nodes [2], [3].

Information theory analyses have proved the benefit of SIC, which relies on successive decoding and subtraction of usersignals, in interference-limited scenarios [4]. SIC attains the corner points of the Gaussian channel's capacity region, and, in contrast to joint detection, decoding complexity is reduced at the exchange of enabling CSI [5], [6]. Hence, the practical application of SIC needs the design of an appropriate power control scheme featuring user-power imbalance, under the specific SIC implementation and energy-aware user constraints.

In the technical literature, we may find a number of practical SIC schemes [6]-[11]. Random spreading codes jointly with a single encoder shared among all users is proposed in [6], [7]. In that respect, system performance strongly depends on the decoder's packet error rate (PER) characteristic. Soft feedback SIC, where the correlator's output is used to perform cancellation, is employed in [8], [9]. This is sometimes inaccurate since estimation errors are propagated along SIC stages. To allow packet error detection, packets are encoded together with cyclic redundancy check (CRC) [10], [11].

Work funded by project TEC2016-76409-C2-1-R (AEI/FEDER, UE) (WINTER): Spanish Ministry of Science, Innovation and Universities, and by the Catalan administration under: (i) 2017 SGR 578; and (ii) the fellowship FI-2018 supported by the Secretary for University and Research of the "Generalitat de Catalunya" and the European Social Fund.
The energy and rate allocation that optimizes the SE of large networks was studied in [10]-[13]. Works [10]-[12] determined the user-asymptotic behaviour of the energy and rate allocation. Therein, variational calculus (VC) constitutes a systematic optimization tool. [12] derived the capacityachieving energy allocation under perfect CSIT at fixed total radiated power. In [10], the optimum energy distribution at the SIC input is obtained for a given encoder with known PER curve shared by all users. Later, [11] extended prior results by considering, instead of a shared encoder, a family of encoders. In [13], the user-energy distribution is optimized at network PER $<10^{-4}$ by a semi-analytic algorithm.

This paper approaches the problem of massive MA under energy-aware constraints, considering aspects of both academical and practical interest. A proof-of-principle scenario, similar to [10], [13], in which a large spread spectrum MA network of users transmitting at constant rate, is analyzed. Moreover, a common central node with strong multiuser decoding potential employing SIC is considered. The scope of this work focuses on designing an energy allocation function, which aided by perfect CSIT and the known PER curve of the employed decoder, maximizes the network SE. In contrast to [10]-[13], system optimization is performed accounting for two per-user energy restrictions: (i) a long-term average energy constraint for energy-aware optimization; (ii) an upper bound to each user's energy, appropriate for low peak-to-average power ratio (PAPR) modulations to set a peak power constraint.

We show that the network SE does not saturate when load regulation activates to avoid the transmission of users with poor channel conditions, in which aspect it differs from the system analyzed in [11]. Both the received energy and SINR profiles are found to be non-increasing functions of the SIC decoding order, while at transmission, the energy allocation function is not monotone. Moreover, the relevance of channel imbalance to network SE is established by comparing, within applicable constraints, the best received energy profile under the most favorable typical log-normal channel with the best channel-independent received energy profile. A chip-level SIC implementation also shows the accuracy of the user-asymptotic model in obtaining the PER evolution over SIC stages.

Section II presents finite-user equations along with the featured SIC scheme and the corresponding user-asymptotic equations. Section III designs the energy allocation tackling SE optimization. Sections IV and V present simulations and conclusions, respectively. 


\section{System Model}

Consider a packet access network comprising a very large population of $K$ low-rate users transmitting towards a common reception point. Transmitted packets comprise a preamble sequence, and payload encoded together with CRC. A common physical layer is adopted for all users, that is, they share the same modulation (low PAPR) and error correcting code of rate $R$. A slotted time division MA, indexed by $m$, is used for system model analysis. At each time slot, users perform two actions before transmission: first, they estimate their local slowly time-varying channel $h[k, m]$ from a regularly broadcast downlink pilot, and, second, they use that information to generate packets with complex symbol energy $E_{\mathrm{x}}[k, m]$. The energy allocation $E_{\mathrm{x}}[k, m]$ is designed to meet the typical peruser energy specifications: the long-term energy constraint (1), and the energy allocation limited to $E_{\max }(2)$ :

$$
\begin{aligned}
\bar{E} & \geq \lim _{m \rightarrow+\infty} \frac{1}{m} \sum_{i=1}^{m} E_{\mathrm{x}}[k, i], \\
E_{\max } & \geq E_{\mathrm{x}}[k, m] .
\end{aligned}
$$

Direct-sequence spread spectrum is adopted for medium access, using the same processing gain $N$ for the random user spreading codes [13]. At every time slot, users are ranked in decreasing order of their individual uplink channel gains $h[1, m]>h[2, m]>\cdots>h[K, m]$. Hence, the specific $m$ index is suppressed, understanding that at each $m$ the user ordering has been modified. At the central node, the aggregate received baseband signal under Gaussian noise $w(t)$ is

$$
y(t)=A[k] \sum_{i=1}^{K} s_{k}(t-\tau[k]) c_{k}(t-\tau[k])+w(t),
$$

with $A[k]$ a complex amplitude factor of magnitude proportional to $\left(E_{\mathrm{x}}[k] h[k]\right)^{1 / 2}$ (assuming $h[k]$ stationary along the packet duration as long as packets are transmitted in agreement with the channel's coherence time), $s_{k}(t)$ and $c_{k}(t)$ the respective data and spreading waveforms of user $k$, and $\tau[k] \approx \tau$ the $k$-th user end-to-end delay according to a quasisynchronous access mode [10], [11]. The signal $y(t)$ is filtered and sampled at the chip-rate.

To counteract MA interference, the key factor limiting network performance, the central node resorts to SIC to recover the $K$ packets transmitted in the same time-frequency resource. In particular, the employed SIC is aided by channel decoder and the CRC error control outputs [10], [11]. Concretely, users are sequentially demodulated. After preamble detection, packet de-spreading and channel decoding, the CRC determines if the packet has been correctly decoded. Thereupon, user-packet parameters are re-estimated using the entire recovered packet, and its waveform is canceled from the input signal [7].

The system model analysis is performed considering two essential univariate functions of the SINR $\Gamma$ known, which characterize imperfect decoding and complex amplitude estimation effects that appear during SIC demodulation [10], [11],
[13]. First, the PER curve of the employed decoder, $\operatorname{PER}[\Gamma]$, and second, the $\varepsilon(\Gamma)$ curve containing the average remaining fraction of uncancelled energy after subtracting each user.

At the $k$-th SIC stage, the SINR $\Gamma[k]$ is determined as a function of the received symbol energy $E_{s}[k] \triangleq E_{\mathrm{x}}[k] h[k]$, and the total noise plus interference term $N_{t}[k]$, as

$$
\Gamma[k]=\frac{E_{s}[k]}{N_{t}[k]}=\frac{E_{s}[k]}{N_{0}+\frac{\theta}{N} \sum_{j<k} \epsilon_{j}(\Gamma[j]) E_{s}[j]+\frac{\theta}{N} \sum_{j>k} E_{s}[k]},
$$

with $N_{0}$ the noise power spectral density (PSD), $0 \leq \theta \leq 1$ an average decorrelation factor associated with timing misalignments, $\frac{1}{N}$ the decorrelation between signature sequences [14], and $\epsilon_{j}(\Gamma)$ a random variable modeling the adopted decodingcancellation scheme, independent and identically distributed over all users [10], [11]. Explicitly, when CRC determines incorrect decoding, which occurs with probability $\operatorname{PER}[\Gamma]$, it equals 1 . Otherwise, it equals $\varepsilon(\Gamma)$ with probability $\operatorname{PSR}[\Gamma] \triangleq$ $1-\operatorname{PER}[\Gamma]$, where PSR $[\Gamma]$ stands for the packet success rate (PSR) function, the PER complementary curve.

\section{A. User-asymptotic system model equations}

Previous works [10]-[12] have shown the convenience of working in the infinite user-regime to analyze large networks. For that reason, we define the continuous user index

$$
t \triangleq \lim _{K \rightarrow+\infty} k / K \quad(0 \leq t \leq 1)
$$

at the same time that $N \rightarrow+\infty$, so that $\alpha \triangleq K / N$, denoting the system load, is asymptotically held constant. First of all, user-asymptotic profiles are defined from previous userfinite variables [10], [11]. Specifically, $k$-indexed variables, represented by the general function $\varphi[k]$, are turned to $\varphi(t)$ with $k=t \cdot K$. Moreover, when channels are identically distributed, the average energy along time (1) turns out to be equivalent to the average energy over the user population. This asymptotically yields

$$
\bar{E} \geq \int_{0}^{1} E_{\mathrm{x}}(t) \mathrm{d} t
$$

Then, as formulated in [10], [11], the user-asymptotic SINR profile $\Gamma(t)$, the ratio between the received symbol energy profile $E_{s}(t)=E_{\mathrm{x}}(t) h(t)$ (with $h(t)$ the decreasing channel profile) and the noise plus interference profile $N_{t}(t)$, is:

$$
\Gamma(t)=\frac{E_{s}(t)}{N_{0}+\alpha \theta \int_{0}^{t} r(\Gamma(\tau)) E_{s}(\tau) \mathrm{d} \tau+\alpha \theta \int_{t}^{1} E_{s}(\tau) \mathrm{d} \tau},
$$

where summations have been turned to integrals with $\mathrm{d} t \triangleq$ $\lim _{K \rightarrow+\infty} 1 / K$, and $r(\Gamma) \triangleq 1-(1-\varepsilon(\Gamma)) \operatorname{PSR}[\Gamma]$ is the average value of the random variable $\epsilon_{j}(\Gamma)$. Equation (7) can be easily manipulated to obtain a differential equation [10], [11]. Its solution states $N_{t}(t)$ to be the following exponential

$$
N_{t}(t)=N_{t}(0) \exp (-B(t)), B(t) \triangleq \alpha \int_{0}^{t} \Phi[\Gamma(\tau)] \mathrm{d} \tau,
$$

with $N_{t}(0)=N_{0}+\alpha \theta \int_{0}^{1} E_{s}(t) \mathrm{d} t$ the initial noise term, and $\Phi[\Gamma] \triangleq \theta(1-\varepsilon(\Gamma)) \Gamma \cdot \operatorname{PSR}[\Gamma]$ a known dependent function of the system decoding-cancellation process. 


\section{EnERgy Allocation Design}

In this Section, we seek to determine the optimum energy allocation $E_{\mathrm{x}}(t)$, as a function of the ordered channel profile $h(t)$, that maximizes the network throughput, which is proportional to the aggregate performance metric

$$
\mathrm{SE}=\frac{1}{1+\beta} \frac{R}{N} \sum_{k=1}^{K} \operatorname{PSR}[\Gamma[k]],
$$

namely, SE, where $\beta$ stands for the roll-off factor of the shaping pulse. The previous SE asymptotically converges to

$$
\mathrm{SE}=\frac{\alpha R}{1+\beta} \int_{0}^{1} \operatorname{PSR}[\Gamma(t)] \mathrm{d} t .
$$

Therefore, the following optimization problem to maximize the network SE, formulated in terms of the symbol energy profile at reception $E_{s}(t)$, under the average energy constraint over the user population (12), the specific SIC (13) taken from (8), and the energy limitation (14), is stated:

$$
\begin{array}{rlrl}
\max _{0<t_{0} \leq 1} & \max _{E_{s}(t)} & & \int_{0}^{t_{0}} \operatorname{PSR}\left[\frac{E_{s}(t)}{N_{t}(t)}\right] \mathrm{d} t \\
\text { s.t. } & \bar{E} & \geq \int_{0}^{t_{0}} \frac{E_{s}(t)}{h(t)} \mathrm{d} t \\
\text { s.t. } & E_{s}(t) & =\Gamma(t) N_{t}(0) \exp (-B(t)) \\
\text { s.t. } & E_{\max } & \geq E_{s}(t) / h(t)
\end{array}
$$

where $0<t_{0} \leq 1$, introduced as an admission control unit that sets $E_{\mathrm{x}}(t)=0$ in $t_{0}<t \leq 1$, is optimized as well. Note that although a real SIC implementation always proceeds in decreasing order of received powers [8], this constraint is obviated understanding that the solution profile $E_{s}(t)$ will be non-increasing in $0 \leq t \leq t_{0}$. This is validated a posteriori in Section IV. Naturally, the solution may depend on the number of binding, or active inequalities, i.e. set as strict equalities. Three cases are distinguished: (i) (14) is active in $0 \leq t \leq t_{0}$; (ii) only (12) is active; and (iii) (12) and part of (14) are active. Previous cases are separately analyzed in the following lines.

\section{A. Case (i). Only (14) is active}

In this case, (12) is obviated, and thus, the solution is

$$
E_{\mathrm{x}}(t)=E_{\max } \text { in } 0 \leq t \leq t_{0} .
$$

That is, a fraction $t_{0}$ of users transmit at maximum energy whereas the rest remain silent. The load regulation parameter $t_{0}$ can be easily found by exhaustive search.

\section{B. Cases (ii) and (iii)}

Both cases are analyzed together. The problem is

$$
\begin{array}{rlrl}
\max _{0<t_{0} \leq 1} & \max _{E_{s}(t)} & & \int_{0}^{t_{0}} \operatorname{PSR}\left[\frac{E_{s}(t)}{N_{t}(t)}\right] \mathrm{d} t \\
\text { s.t. } & \bar{E} & =\int_{0}^{t_{0}} \frac{E_{s}(t)}{h(t)} \mathrm{d} t \\
\text { s.t. } & E_{s}(t) & =\Gamma(t) N_{t}(0) \exp (-B(t)) \\
\text { s.t. } & E_{\max } & \geq E_{s}(t) / h(t)
\end{array}
$$

where (12) is set with equality. Therefore, (16-19) constitutes two optimization problems. The inner problem is, for a fixed $t_{0}$ value, a VC problem under $E_{s}(t)$, and the outer problem is found to be univariate under $t_{0}$. Both explicit derivations are moved to the Appendix for clarity of explanation.

The solution of the inner VC is cast in terms of the SINR profile $\Gamma_{0}(t)$ in $0 \leq t \leq t_{0}$ that follows

$$
\lambda=\frac{\operatorname{PSR}^{\prime}\left[\Gamma_{0}(t)\right]}{N_{t}(t)\left(\frac{\beta(t)}{h(t)}+\rho\right)-\alpha\left(I_{\mathrm{x}}(t)+\rho I(t)\right) \Phi^{\prime}\left[\Gamma_{0}(t)\right]},
$$

with $I_{\mathrm{x}}(t) \triangleq \int_{t}^{t_{0}} \beta(\tau) E_{\mathrm{x}}(\tau) \mathrm{d} \tau, I(t) \triangleq \int_{t}^{t_{0}} E_{s}(\tau) \mathrm{d} \tau$, and $\rho \triangleq$ $\alpha \theta I_{\mathrm{x}}(0) / N_{0}$ an auxiliary variable. Moreover, $\lambda>0$ is a scalar to be determined in order to set the energy constraint (17), and $\beta(t) \geq 1$ is a function that satisfies

$$
(\beta(t)-1)\left(E_{\mathrm{x}}(t)-E_{\max }\right)=0 .
$$

In addition, when $t_{0}<1$ is enabled, the following is verified

$$
\frac{\operatorname{PSR}^{\prime}\left[\Gamma_{0}\left(t_{0}\right)\right] \Gamma_{0}\left(t_{0}\right)}{\operatorname{PSR}\left[\Gamma_{0}\left(t_{0}\right)\right]}=\frac{\beta\left(t_{0}\right)+\rho h\left(t_{0}\right)}{1+\rho h\left(t_{0}\right)} .
$$

In particular, in absence of energy limitation, the last user SINR, $\Gamma^{*}=\Gamma_{0}\left(t_{0}\right)$, is assigned to a concrete point that only depends on the PER characteristic of the adopted decoder through $\operatorname{PSR}^{\prime}\left[\Gamma^{*}\right] \Gamma^{*}=\operatorname{PSR}\left[\Gamma^{*}\right]$, with $\Gamma^{*}$ located at the right of the inflexion point of $\operatorname{PSR}[\Gamma]$.

Solution procedure: As typically performed when inequality constraints are considered, the solution needs to be computed for every case, which are described in the sequel.

1) Inequalities in (19) do not hold: In this case, $\beta(t)=1$ in $0 \leq t \leq t_{0}$, and $I_{\mathrm{x}}(0)=\bar{E}$. Hence, (20) is simplified as

$$
\lambda=\frac{\operatorname{PSR}^{\prime}\left[\Gamma_{0}(t)\right]}{N_{t}(t)\left(\frac{1}{h(t)}+\frac{\alpha \theta \bar{E}}{N_{0}}\right)-\alpha\left(I_{\mathrm{x}}(t)+\frac{\alpha \theta \bar{E}}{N_{0}} I(t)\right) \Phi^{\prime}\left[\Gamma_{0}(t)\right]} .
$$

The solution can be obtained by solving (23) jointly with (18) for temporarily known $\lambda>0$ and $I(0)>0$. Exhaustive searches over $\lambda, I(0)$ need to be performed, so as to fulfil

$$
\bar{E}=\int_{0}^{t_{0}} E_{\mathrm{x}}(t) \mathrm{d} t, I(0)=\int_{0}^{t_{0}} E_{s}(t) \mathrm{d} t .
$$

For the cases whenever the solution of (23) does not satisfy (19), some values of $E_{\mathrm{x}}(t)$ need to be set $E_{\max }$.

2) A set of inequalities in (19) is active: How to determine which set of values are active is solved in the following lines taking as reference the stationary point equation (20). Note that in this case, the tuple $\lambda, I(0), I_{\mathrm{x}}(0)$ is required to be obtained by exhaustive search, keeping $\Gamma_{0}(t)$ candidates that simultaneously fulfil (24) and the additional equation

$$
I_{\mathrm{x}}(0)=\int_{0}^{t_{0}} \beta(t) E_{\mathrm{x}}(t) \mathrm{d} t \geq \bar{E} .
$$

It is straightforward to see that at every $t$, the unknown is: (i) $\Gamma_{0}(t)$ when $E_{\mathrm{x}}(t)<E_{\max }$, or (ii) $\beta(t)$ when $E_{\mathrm{x}}(t)=E_{\max }$. The solution is obtained from (20) as previously described while monitoring $E_{\mathrm{x}}(t)$. If at some $t$, (19) is violated, enforce $E_{\mathrm{x}}(t)=E_{\max }$, and find such $\beta(t)$ that fulfils (20). Afterwards, at the next $t$, repeat the aforementioned procedure. 


\section{NUMERICAL RESULTS}

A scenario is envisaged where users are subject, over time, to the same channel statistics. Thus, over the same access slot, independent users perceive all channel realizations in accordance with the given distribution. The channel profile $h(t)$ is obtained taking as reference the log-normal probability density function $f_{H}(h ; \mu, \sigma)$, upper-bounded by $h_{\max }=10$. Then, $h(t)$ is computed from the cumulative distribution function $F_{\widetilde{H}}(h)$, as $h(t) \triangleq F_{\widetilde{H}}^{-1}(1-t)$ :

$$
F_{\widetilde{H}}(h)=\left(\int_{0}^{h} f_{H}(x) \mathrm{d} x\right)\left(\int_{0}^{h_{\max }} f_{H}(x) \mathrm{d} x\right)^{-1} h \leq h_{\max } .
$$

For a fair comparison, $\mu$ is adjusted to set $h(t)$ to unit-mean. $\theta$ is set to 1 [10]. The standardized turbo code (TC) of rate $1 / 2$ with QPSK modulation (low PAPR, $R=1 \mathrm{bps} / \mathrm{Hz}$ ), and packets of 48 preamble symbols and 440 information bits is adopted [15]. At reception, the channel decoder operates under 10 max-log-map iterations. Due to lack of space, PER $[\Gamma]$ and $\varepsilon(\Gamma)$ are not depicted. Simulations carried out show that $\Gamma^{*}=$ 1.47 , with $\operatorname{PER}\left[\Gamma^{*}\right] \approx 0.04$ and $\varepsilon\left(\Gamma^{*}\right) \approx 0.0014$.

Our analysis is twofold. Firstly, the case of having no $E_{\max }$ constraint is analyzed, that is, $E_{\max }>\max _{t} E_{\mathrm{x}}(t)$. Secondly, $E_{\max }=\max _{t} E_{\mathrm{x}}(t)$ is studied. Both cases, namely 1 and 2 , are respectively analyzed in Sections IV-A and IV-B.

\section{A. Case 1. $E_{\max }>\max _{t} E_{\mathrm{x}}(t)$}

Fig. 1 depicts the normalized throughput $\alpha \int_{0}^{t_{0}} \operatorname{PSR}\left[\Gamma_{0}(t)\right] \mathrm{d} t$ as a function of the system traffic load for the working point $\bar{E} / N_{0}=8 \mathrm{~dB}$, and the channel parameter $\sigma=0.30$.

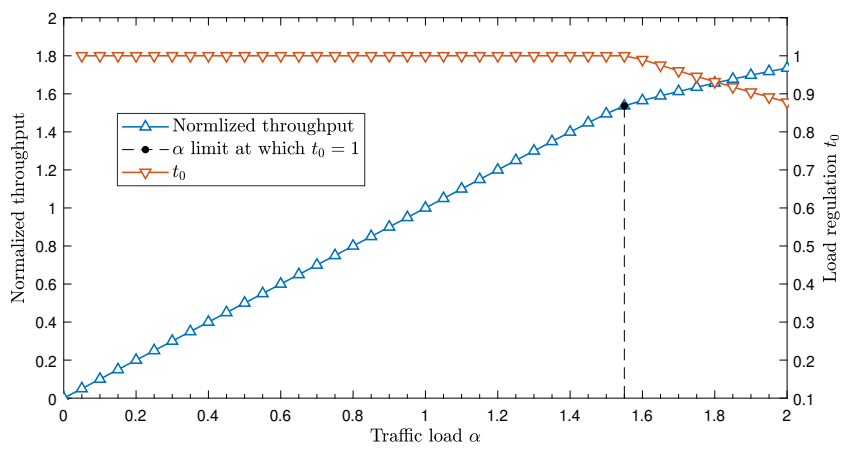

Fig. 1. Normalized throughput (left axis) and $t_{0}$ (right axis) vs. $\alpha$.

In blue markers, the normalized throughput is depicted, which increases practically linearly in $\alpha$ at $\alpha<1.55$. In this regime, it is beneficial to allocate energy to all users, instead of enable $t_{0}<1$ and assign energy to those active. This is because, in the latter, although increase the energy transference, strongly penalizes system throughput. At $\alpha=1.55$, the limit value at which $t_{0}=1$, the normalized throughput is 1.54 . At $\alpha>1.55, t_{0}<1$, depicted using red markers, operates to set $\Gamma_{0}\left(t_{0}\right)=\Gamma^{*}$ without saturating the network throughput. This contrasts with prior works where load regulation acts to set $\alpha t_{0}$ constant, thus, saturating the network throughput [11]. Here, instead, load $\alpha\left(1-t_{0}\right)$ associated to users with poor channel gains $h\left(t_{0}<t \leq 1\right)$ remain silent, whereas the active load $\alpha t_{0}$ achieves throughput values increasing in $\alpha$.
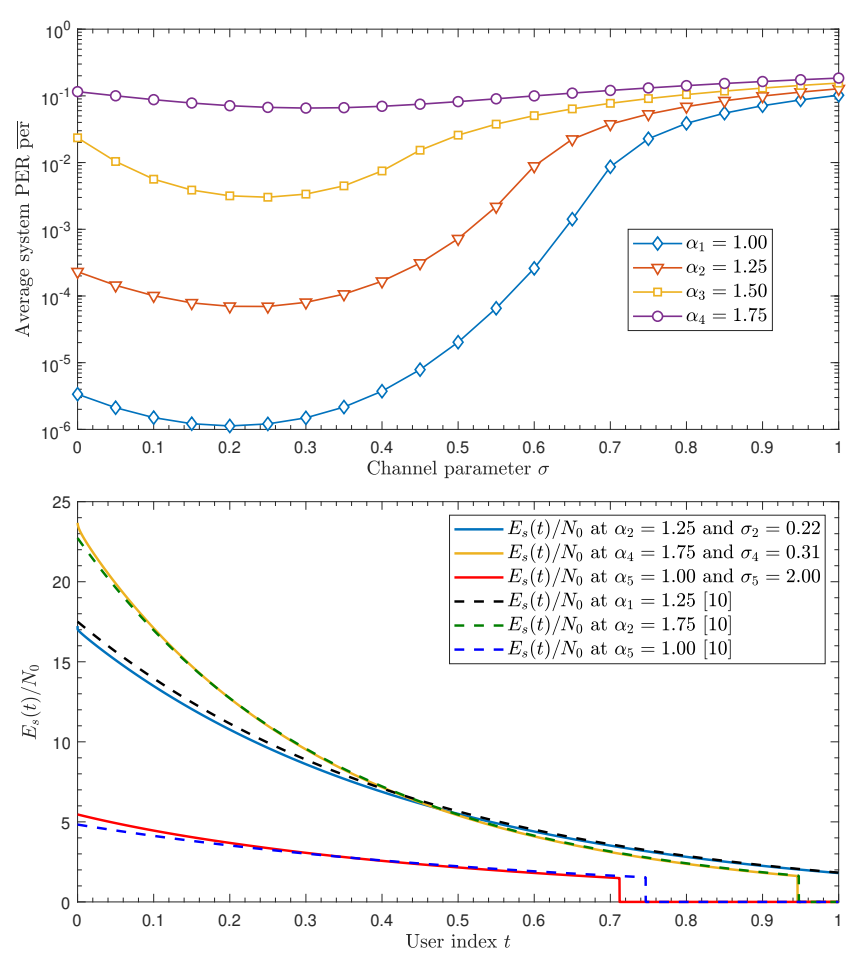

Fig. 2. In the upper part: $\overline{\text { per }}$ vs. $\sigma$ for $\alpha_{1}=1.00, \alpha_{2}=1.25$, $\alpha_{3}=1.50$, and $\alpha_{4}=1.75$. In the lower part, the optimum $E_{s}(t) / N_{0}$ : (i) for our design (solid lines), and (ii) for the channel-independent design optimizing at the SIC input (dashed lines) [10].

Secondly, in the upper part of Fig. 2, the average system $\mathrm{PER}, \overline{\mathrm{per}} \triangleq \int_{0}^{t_{0}} \operatorname{PER}\left[\Gamma_{0}(t)\right] \mathrm{d} t$, versus the channel parameter $\sigma$ is analyzed for different $\alpha$ values. As shown, the energy allocation takes benefit of channel unbalance up to a certain $\sigma$ value, after which no more energy is available to compensate worse user-channels. This benefit, measured in terms of $\overline{\text { per, }}$ results relevant (less significant) at low (high) $\alpha$. In particular, the most favorable $\sigma$ at each load are found to be $\sigma_{1}=0.20$, $\sigma_{2}=0.22, \sigma_{3}=0.24$, and $\sigma_{4}=0.31$, respectively.

In the lower part of Fig. 2, the symbol energy over noise PSD (EsNo) profiles at reception $E_{s}(t) / N_{0}$, from the proposed system optimization (solid), are compared with the best channel-independent EsNo profiles at the SIC input (dashed) [10] for the same received average EsNo $I(0) / N_{0}$. Certainly, the optimum $E_{\mathrm{x}}(t)$ is such that generates $E_{s}(t)=E_{\mathrm{x}}(t) h(t)$ similar to the SE-maximizer profile at reception [10]. In fact, the generated $E_{s}(t)$ maximizes the energy transference without severely penalizing SE. Also, $E_{s}(t)$ are shown to be non-increasing, which validates the hypothesis in Section III.

Finally, a chip-level SIC implementation is simulated to validate the large system analysis proposed herein. Fig. 3 depicts the asymptotic (black) and empirical (red, blue and yellow) PER profiles associated with two points of Fig. 1. In particular, the traffic loads 1.40 and 1.75 are evaluated. Empirical PER curves are obtained for a chip-synchronous simulation where users are uniformly sampled from the obtained optimum $E_{s}(t)$ profiles. Different processing gains $N$ are evaluated while setting the chip rate at $1.92 \mathrm{Mcps}$. A square root raised cosine filter with $\beta=0.35$ is used [15]. 


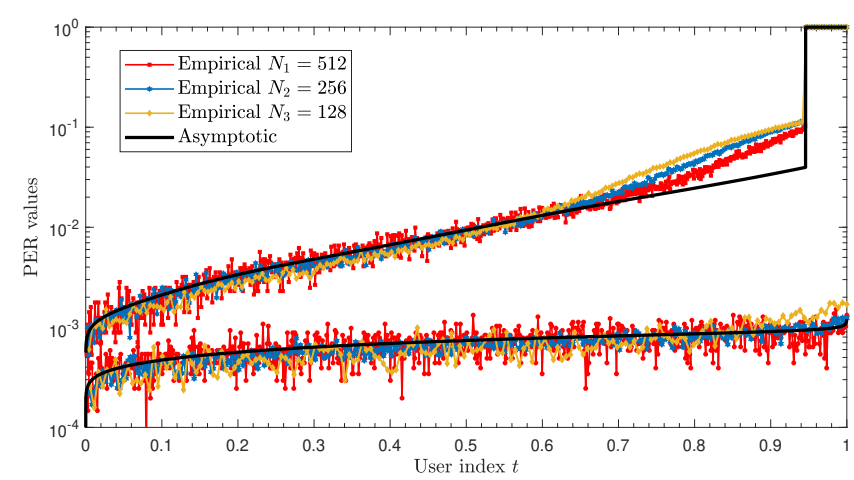

Fig. 3. Asymptotic and empirical PER profiles: (i) $\alpha=1.40$ (lower curve); and (ii) $\alpha=1.75$ (upper curve) with $t_{0} \approx 0.95$. Empirical profiles are obtained for $N_{1}=512, N_{2}=256$, and $N_{3}=128$, which respectively result in: (i) $K_{1}=717, K_{2}=358$ and $K_{3}=179$ users; and (ii) $K_{1}=896, K_{2}=448$ and $K_{3}=224$ users.

Empirical curves approach the user-asymptotic one as $N$ increases. At finite $N$, some PER discrepancies affect the last users of the SIC decoding order, an effect mitigated as $N$ increases. This appears due to user-finite behavior, which results negligible at the first SIC decoding stages and becomes relevant in last SIC decoding stages. The mentioned effect is highlighted as the activity load approaches the limit traffic load $\alpha=1.55$ at which load regulation $t_{0}$ starts. This differs from [10] where high level, instead of chip level, Monte Carlo simulations evidenced similar user-PER mismatching. Authors in [13] also obtained miss-agreements between large and moderate $N$ values in SIC measurements.

\section{B. Case 2. $E_{\max }=\max _{t} E_{\mathrm{x}}(t)$}

This Section is devoted to evaluating the impact of $E_{\max }$ over the solution profiles. We depict in Fig. 4, for the scenario parameters described at the beginning of Section IV, the energy profiles at transmission, $E_{\mathrm{x}}(t)$, for two traffic loads.

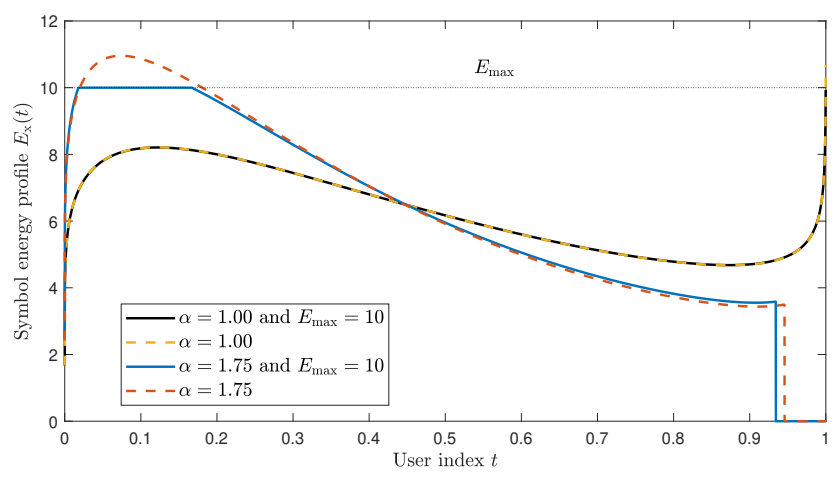

Fig. 4. Optimum $E_{\mathrm{x}}(t)$ profiles at $\alpha=1.00$, and $\alpha=1.75$ when $E_{\max }$ does not apply (dashed lines), and $E_{\max }=10$ (solid lines).

First, $E_{\mathrm{x}}(t)$ are shown not to be, necessarily, monotone functions of the user-variable $t$. When maximum energy limitation applies, the respective energy profiles $E_{\mathrm{x}}(t)$ are upperbounded by $E_{\max }$ near those $t$-values which initially violate that constraint. Moreover, $E_{\max }$ may pose some limitations to network performance, specially so, under high activity loads. At $\alpha=1.75$, the normalized throughput and the average PER are degraded by $1.7 \%$ and $24 \%$, respectively.

\section{CONCLUSIONS}

The network spectral efficiency optimization of a massive spread spectrum multiple access network, where users operate under an energy-driven allocation scheme and a successive interference cancellation central node, has been addressed in this work. An energy allocation design based on perfect channel knowledge at transmission and per-user energyaware constraints has been undertaken. Specifically, a longterm average energy constraint, and, an energy limitation appropriate for low peak-to-average power ratio modulations have been considered. For system optimization, both the PER characteristic of the employed decoder and the known SIC decoding-cancellation model are employed, in combination with variational calculus, to determine the user-asymptotic energy allocation that maximizes network spectral efficiency. We have shown that a non-monotone allocation function maximizes this problem, whereas at the SIC input, the energy distribution profile is found to be a non-increasing function. The user-asymptotic analysis and associated system optimization have been validated by exhaustive Monte Carlo simulations at chip-level and employing a standardized encoder.

\section{APPENDIX}

The present Section is devoted to the resolution of (16-18). We first substitute (18) in (17) and (19) to seek, instead of $E_{s}(t), \Gamma(t)$ in $0 \leq t \leq t_{0}$. Now, we have:

$$
\begin{aligned}
\max _{0<t_{0} \leq 1} \max _{\Gamma(t)} & \int_{0}^{t_{0}} \operatorname{PSR}[\Gamma(t)] \mathrm{d} t \\
\text { s.t. } \bar{E} & =\int_{0}^{t_{0}} \frac{\Gamma(t)}{h(t)} N_{t}(0) e^{-B(t)} \mathrm{d} t \\
\text { s.t. } & E_{\max } \geq \frac{\Gamma(t)}{h(t)} N_{t}(0) e^{-B(t)}
\end{aligned}
$$

where the optimum $\Gamma(t)$ can be found using VC tools [16]. We consider variations $\Gamma(t)=\Gamma_{0}(t)+a \cdot \phi(t)$ in $0 \leq t \leq t_{0}$, where $a$ and $\phi(t)$ denote the magnitude and direction of those variations around any $\Gamma_{0}(t)$. The Lagrangian of (27-29) is

$$
\begin{aligned}
\mathcal{L} & \triangleq \int_{0}^{t_{0}} \operatorname{PSR}[\Gamma(t)] \mathrm{d} t-\lambda\left(\int_{0}^{t_{0}} \frac{\Gamma(t)}{h(t)} N_{t}(0) e^{-B(t)} \mathrm{d} t-\bar{E}\right) \\
& -\int_{0}^{t_{0}} \mu(t)\left(\frac{\Gamma(t)}{h(t)} N_{t}(0) e^{-B(t)}-E_{\max }\right) \mathrm{d} t,
\end{aligned}
$$

where (28) is introduced using the scalar $\lambda$, and (29) utilizing the function $\mu(t) \geq 0$ only active at those $t$ where (29) is set with equality. The Lagrangian can be simplified by introducing $\beta(t) \triangleq 1+\mu(t) / \lambda \geq 1$, and obviating additive terms independent of $\Gamma(t)$, we have

$$
\tilde{\mathcal{L}}=\int_{0}^{t_{0}} \operatorname{PSR}[\Gamma(t)] \mathrm{d} t-\lambda \int_{0}^{t_{0}} \beta(t) \frac{\Gamma(t)}{h(t)} N_{t}(0) e^{-B(t)} \mathrm{d} t .
$$

Before continuing, we define the auxiliary functions

$$
I_{\mathrm{x}}(t) \triangleq \int_{t}^{t_{0}} \beta(\tau) E_{\mathrm{x}}(\tau) \mathrm{d} \tau, I(t) \triangleq \int_{t}^{t_{0}} E_{s}(\tau) \mathrm{d} \tau
$$


As stated in [16], the optimum $\Gamma_{0}(t)$ is such that the gradient under variable $a$ when led to zero, $\nabla_{a \rightarrow 0} \tilde{\mathcal{L}}$, vanishes. The contribution of the first term in (31) to $\nabla_{a \rightarrow 0} \tilde{\mathcal{L}}$ yields

$$
\int_{0}^{t_{0}} \operatorname{PSR}^{\prime}\left[\Gamma_{0}(t)\right] \phi(t) \mathrm{d} t \text {. }
$$

The gradient over the second term is, applying the product differentiation rule to the factor of $\beta(t)$ in (31)

$$
\begin{aligned}
& \lambda \int_{0}^{t_{0}} \beta(t) \frac{N_{t}(t)}{h(t)} \phi(t) \mathrm{d} t+\lambda I_{\mathrm{x}}(0) \frac{\nabla_{a \rightarrow 0} N_{t}(0)}{N_{t}(0)} \\
& -\alpha \lambda \int_{0}^{t_{0}} \beta(t) E_{\mathrm{x}}(t)\left(\int_{0}^{t} \Phi^{\prime}\left[\Gamma_{0}(\tau)\right] \phi(\tau) \mathrm{d} \tau\right) \mathrm{d} t .
\end{aligned}
$$

The second term is computed in Appendix-B, whereas the third term is integrated by parts with $u \triangleq \int_{0}^{t} \Phi^{\prime}\left[\Gamma_{0}(\tau)\right] \phi(\tau) \mathrm{d} \tau$ and $d v \triangleq \beta(t) E_{\mathrm{x}}(t) \mathrm{d} t$, obtaining $\int_{0}^{t_{0}} I_{\mathrm{x}}(t) \Phi^{\prime}\left[\Gamma_{0}(t)\right] \phi(t) \mathrm{d} t$. Substituting both results into (34), we finally get the contribution of the second term in (31) to $\nabla_{a \rightarrow 0} \tilde{\mathcal{L}}$

$$
\lambda \int_{0}^{t_{0}} D(t) \phi(t) \mathrm{d} t
$$

where $D(t) \triangleq N_{t}(t)\left(\frac{\beta(t)}{h(t)}+\rho\right)-\alpha\left(I_{\mathrm{x}}(t)+\rho I(t)\right) \Phi^{\prime}\left[\Gamma_{0}(t)\right]$, and $\rho \triangleq \alpha \theta I_{\mathrm{x}}(0) / N_{0}$. Equation (35), together with (33), yields

$$
\int_{0}^{t_{0}}\left(\operatorname{PSR}^{\prime}\left[\Gamma_{0}(t)\right]-\lambda D(t)\right) \phi(t) \mathrm{d} t=0,
$$

which is set to zero, and, invoking the Fundamental Lemma of the Calculus of Variations [16], it needs to be zero for any admissible $\phi(t)$. This implies $\operatorname{PSR}^{\prime}\left[\Gamma_{0}(t)\right]-\lambda D(t)=0$ in $0 \leq t \leq t_{0}$. Thus, we finally get the stationary point equation

$$
\lambda=\frac{\operatorname{PSR}^{\prime}\left[\Gamma_{0}(t)\right]}{N_{t}(t)\left(\frac{\beta(t)}{h(t)}+\rho\right)-\alpha\left(I_{\mathrm{x}}(t)+\rho I(t)\right) \Phi^{\prime}\left[\Gamma_{0}(t)\right]} .
$$

\section{A. Optimum load regulation parameter $t_{0}$}

The outer optimization problem (27-29) to obtain the optimum $t_{0}<1$, when enabled, is computed in the sequel. Specifically, two simultaneous equations must be satisfied. First, the derivative of (30) w.r.t. $t_{0}$ is set to zero. This gives

$$
\operatorname{PSR}\left[\Gamma_{0}\left(t_{0}\right)\right]=\lambda\left(E_{\mathrm{x}}\left(t_{0}\right)+I_{\mathrm{x}}(0) \frac{\nabla_{t_{0}} N_{t}(0)}{N_{t}(0)}\right),
$$

with $\nabla_{t_{0}} N_{t}(0)=\left(\alpha \theta / N_{0}\right) \cdot N_{t}(0) E_{\mathrm{x}}\left(t_{0}\right) h\left(t_{0}\right)$ computed differentiating (42). Substituting it into (38), we get

$$
\operatorname{PSR}\left[\Gamma_{0}\left(t_{0}\right)\right]=\lambda E_{\mathrm{x}}\left(t_{0}\right)\left(1+\rho h\left(t_{0}\right)\right) .
$$

Secondly, at $t=t_{0}$, the stationary point equation (37) gives

$$
\operatorname{PSR}^{\prime}\left[\Gamma_{0}\left(t_{0}\right)\right]=\lambda N_{t}\left(t_{0}\right)\left(\beta\left(t_{0}\right) h^{-1}\left(t_{0}\right)+\rho\right) .
$$

After some straightforward manipulations, we divide both equations to finally obtain the equation that optimum load regulation must satisfy:

$$
\frac{\operatorname{PSR}^{\prime}\left[\Gamma_{0}\left(t_{0}\right)\right] \Gamma_{0}\left(t_{0}\right)}{\operatorname{PSR}\left[\Gamma_{0}\left(t_{0}\right)\right]}=\frac{\beta\left(t_{0}\right)+\rho h\left(t_{0}\right)}{1+\rho h\left(t_{0}\right)} .
$$

In particular, when no energy limitation is considered, $\beta\left(t_{0}\right)=$ 1 , and thus, $\operatorname{PSR}^{\prime}\left[\Gamma_{0}\left(t_{0}\right)\right] \Gamma_{0}\left(t_{0}\right)=\operatorname{PSR}\left[\Gamma_{0}\left(t_{0}\right)\right]$.

\section{B. Auxiliary computation}

$$
\begin{aligned}
& \text { From } N_{t}(0)=N_{0}+\alpha \theta \int_{0}^{t_{0}} \Gamma(t) N_{t}(0) e^{-B(t)} \mathrm{d} t \\
& \qquad N_{t}(0)=N_{0}\left(1-\alpha \theta \int_{0}^{t_{0}} \Gamma(t) e^{-B(t)} \mathrm{d} t\right)^{-1}
\end{aligned}
$$

$\nabla_{a \rightarrow 0} N_{t}(0)$ is, applying the product rule over $\Gamma(t) e^{-B(t)}$

$$
\frac{\alpha \theta}{N_{0}} N_{t}(0) \int_{0}^{t_{0}}\left(N_{t}(t) \phi(t)-\alpha E_{s}(t) \int_{0}^{t} \Phi^{\prime}\left[\Gamma_{0}(\tau)\right] \phi(\tau) \mathrm{d} \tau\right) \mathrm{d} t .
$$

Now, integrating by parts with $u \triangleq \int_{0}^{t} \Phi^{\prime}\left[\Gamma_{0}(\tau)\right] \phi(\tau) \mathrm{d} \tau$ and $d v \triangleq E_{s}(t) \mathrm{d} t$, we get

$$
\frac{\nabla_{a \rightarrow 0} N_{t}(0)}{N_{t}(0)}=\frac{\alpha \theta}{N_{0}} \int_{0}^{t_{0}}\left(N_{t}(t)-\alpha I(t) \Phi^{\prime}\left[\Gamma_{0}(t)\right]\right) \phi(t) \mathrm{d} t \text {. }
$$

\section{REFERENCES}

[1] S. Lien, K. Chen, and Y. Lin, "Toward ubiquitous massive accesses in 3GPP machine-to-machine communications," IEEE Commun. Mag., vol. 49, no. 4, pp. 66-74, April 2011.

[2] H. S. Dhillon, H. Huang, H. Viswanathan, and R. A. Valenzuela, "Fundamentals of throughput maximization with random arrivals for M2M communications," IEEE Trans. Commun., vol. 62, no. 11, pp. 4094-4109, November 2014.

[3] Z. Ding, X. Lei, G. K. Karagiannidis, R. Schober, J. Yuan, and V. K. Bhargava, "A survey on non-orthogonal multiple access for $5 \mathrm{G}$ networks: Research challenges and future trends," IEEE J. Sel. Areas Commun., vol. 35, no. 10, pp. 2181-2195, October 2017.

[4] S. P. Weber, J. G. Andrews, X. Yang, and G. de Veciana, "Transmission capacity of wireless ad hoc networks with successive interference cancellation," IEEE Trans. Info. Theory, vol. 53, no. 8, pp. 2799-2814, August 2007.

[5] Y. Zhang, K. Peng, Z. Chen, and J. Song, "SIC vs. JD: Uplink NOMA techniques for M2M random access," in 2017 IEEE Int. Conference on Communications (ICC), May 2017, pp. 1-6.

[6] G. Gallinaro, N. Alagha, R. De Gaudenzi, K. Kansanen, R. Müller, and P. S. Rossi, "ME-SSA: An advanced random access for the satellite return channel," in 2015 IEEE Int. Conference on Communications (ICC), June 2015, pp. 856-861.

[7] R. De Gaudenzi, O. Del Río Herrero, and G. Gallinaro, "Enhanced spread aloha physical layer design and performance," Int. Journal on Satellite Communications and Networking, vol. 32, no. 6, pp. 457-473, 2014.

[8] M. Mohammad and R. M. Buehrer, "The effects of ordering criteria in linear successive interference cancellation in CDMA systems," IEEE Trans. Wireless Commun., vol. 7, no. 11, pp. 4128-4132, Nov. 2008.

[9] F. Berggren and S. Ben Slimane, "Power allocation for a simple successive interference cancellation scheme in a multi-rate DS-CDMA system," in 2002 IEEE Int. Conference on Communications (ICC), vol. 1, April 2002, pp. 351-355.

[10] J. Sala-Álvarez, F. Rey, J. Villares, and F. Molina, "Minimum PER user-energy profile for massive SIC receivers under an average energy constraint," in 2017 IEEE 18th Int. Workshop on Signal Processing Advances in Wireless Communications (SPAWC), July 2017, pp. 1-6.

[11] F. Molina, J. Sala-Álvarez, J. Villares, and F. Rey, "Joint energy and rate allocation for successive interference cancellation in the finite blocklength regime," in 2018 6th IEEE Global Conference on Signal and Information Processing (GlobalSIP), September 2018, pp. 1-5.

[12] D. V. Djonin and V. K. Bhargava, "Asymptotic analysis of the conventional decision feedback receiver in fading channels," IEEE Trans. Wireless Commun., vol. 2, no. 5, pp. 1066-1078, September 2003.

[13] F. Collard and R. De Gaudenzi, "On the optimum packet power distribution for spread aloha packet detectors with iterative successive interference cancelation," IEEE Trans. Wireless Commun., vol. 13, no. 12, pp. 6783-6794, December 2014.

[14] S. Verdú and S. Shamai (Shitz), "Spectral efficiency of CDMA with random spreading," IEEE Trans. Inf. Theory, vol. 45, no. 2, pp. 622640, March 1999.

[15] EN ETSI, "Digital video broadcasting (DVB); interaction channel for satellite distribution systems," ETSI EN 301790 (V1.4.1), 2005.

[16] I. M. Gelfand, R. A. Silverman et al., Calculus of variations. Courier Corporation, 2000. 